\title{
Efficient Mobile Sensor Authentication In Smart Home and WPAN
}

\author{
Kyusuk Han, Taeshik Shon, Member, IEEE, and Kwangjo Kim, Member, IEEE
}

\begin{abstract}
Currently, it is rapidly increasing convergence services based on various mobile devices with sensors like Smart Home. Specifically the mobility of the sensors in Smart Home merged with wireless sensor networks (WSN) brings security issues such as re-authentication and tracing the node movement. We extend our novel and efficient node authentication and key exchange protocol that support Irregular distribution. Compared with previous protocols, our protocol has only a third of communication and computational overhead. We expect our protocol to be the efficient solution that increases the lifetime of sensor network ${ }^{l}$.
\end{abstract}

Index Terms - Wireless Sensor Networks, Authentication, Mobile node, Untraceability, Key Distribution.

\section{INTRODUCTION}

Wireless Sensor Network (WSN) is the network that consists of lightweight devices with short-ranged wireless communication and battery-powered. The devices have the sensor that gathers the environmental information and etc. After sensing this information, the devices send the information to the networks. The recent advance made the WSN technologies be applied in various areas such as Smart Digital Home Network [13]-[14], Wireless Personal Area Network (WPAN) and Wireless Sensor and Actor Network (WSAN) [7]-[8]. Recently, RF4CE also deploy Zigbee (IEEE 802.15.4) [11] as underlying communication technologies, which is designed to substitute the current IR communication. In such environments, handling a large overhead from frequent node re-authentication requests due to the continuous node movements and the threats of tracing the node movement are important security issues.

While most security researches on the WSN remain on how to efficiently utilize the limited resources in static network environments $[1,5,9,10,12]$, a few researches begin to consider the security in the dynamic environments. Reference [6] argued the possible presence of mobile node, and proposed the authentication protocol supporting node mobility that does not require any sink or base station for authentication and key distribution. Their model requires the large communication and computation cost when the node is continuously moved

$\mathrm{K}$. Han is with the department of Computer Science, KAIST, Daejeon, Korea (e-mail :hankyusuk@kaist.ac.kr).

Corresponding Author: T. Shon is with Convergence Solution Team, Digital Media \& Communication R\&D Center, Samsung Electronics, Suwon, Korea (e-mail : ts.shon@samsung.com).

Corresponding Author : K. Kim is with the department of Computer Science, KAIST, Daejeon, Korea (e-mail :kkj@kaist.ac.kr). though. In order to minimize such overhead, we proposed efficient node authentication and key exchange model that reduces communication and computational costs for node reauthentication and also provides untraceablity to mobile nodes [2]. In the model, once a mobile node is firstly authenticated by a static sink, the node can be efficiently authenticated by the neighbor sinks of the firstly connected sink.

However, the previous model has the limit that the protocol may not properly work in the environment that the sensors are irregularly distributed. In case of the smart home, the electric devices that attach sensors may be distributed irregularly as in Fig. 1. In such environment, the remote controller may fail to be re-authenticated depending on the node movements.

Therefore, our motivation is to provide the improved node authentication and key exchange model suitable for such irregularly distribution. Applying our improvement, the mobile node can be authenticated by the sink that is not the neighbor of the formerly connected sink.

The paper organized as follows: Section II describes the mobility of the sensor network and the previous authentication and key exchange protocol. We argue the problem in the irregularly distributed environments and show the improved protocol in Section III. Section IV shows the analysis of the protocol, and Section $\mathrm{V}$ concludes this paper.

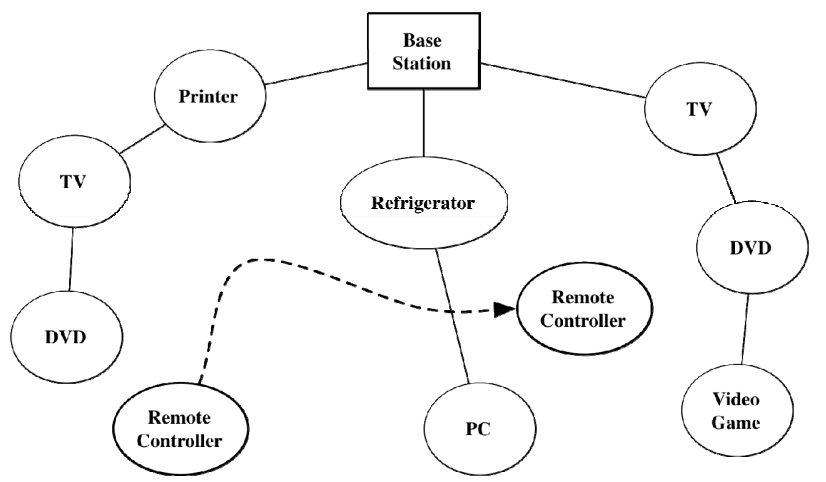

Fig. 1 RF4CE deploys Zigbee based sensor network technologies as underlying communication technology. In such environment, the sensor in the remote controller has the mobility.

\section{Problems in The SMart Home}

In this section, we describe the brief procedure of original protocol, and claim problems applying to the smart home.

\section{A. Overall Process of Previous Protocol}

The overall protocol is divided in to five phases: Periodical Neighbor Discovery (Phase 0), Neighbor Sink Setup (Phase 1), Neighbor Group Key Distribution (Phase 2), Node Initial 
Authentication (Phase 3), and Node Re-authentication (Phase 4).

Assume that there are a base station $B S$, a sink $S_{l}$, a neighbor sink $S_{2}$, and a mobile node $N$ in the network. We define the neighbor sink as the sink that is in the 1 hop communication range.

During phase 0 , every sink such as $S_{1}$ and $S_{2}$ periodically broadcasts HELLO. If no attempt happens, phase 0 is just discarded.

When $S_{2}$ receives HELLO from $S_{1}, S_{2}$ initiates the neighbor relationship if $S_{l}$ is a newly discovered sink. After the pairwise key between $S_{I}$ and $S_{2}$ has been exchanged in phase $1, S_{I}$ and $S_{2}$ exchange the authentication key that is used to verify the authenticated user in phase 2. Phase 1 and phase 2 are only required during establishing the static sensor network. We let the establishing the static sensor network follows the any previous protocol such as [4].

When $N$ firstly joins the network, $N$ may be connected to $S_{I}$ in the network as in Fig. 2. After receiving HELLO of $S_{l}, N$ initiates the initial authentication with $S_{I}$ in Phase 3. Once $N$ is authenticated $S_{l}, N$ only needs the re-authentication in Phase 4 when $N$ continuously moves and request the authentication again. The authentication process in Phase 3 is only necessary when the re-authentication fails due to the certain case that the neighbor sink is not available.

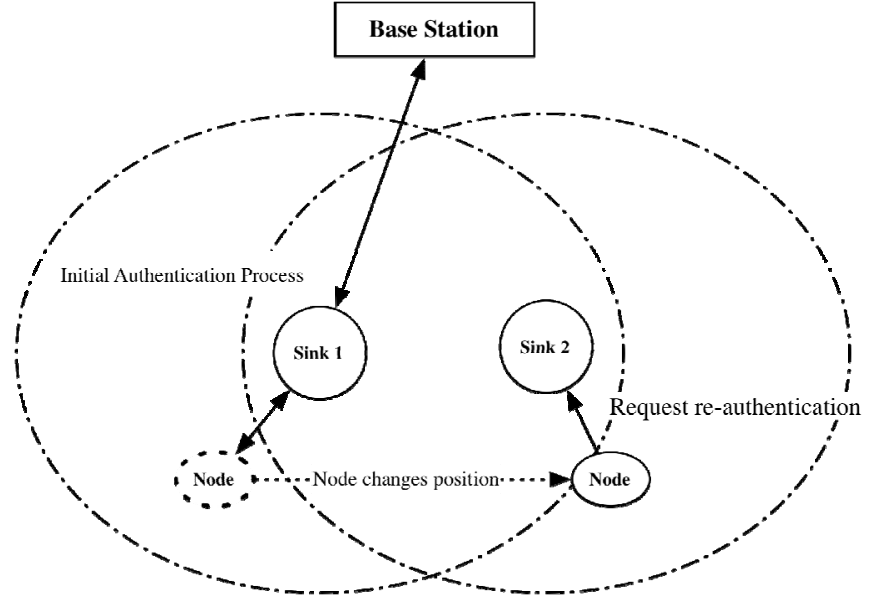

Fig. 2 The base station is only involved when the sink 1 firstly authenticates the node (Phase 3). Next time, the node is directly authenticated by sink 2 without the base station (Phase 4).

\section{B. Authentication Ticket}

The previous protocol introduced the Authentication Ticket that is the proof of the node is authenticated. A node receives the authentication ticket from the sinks during the authentication process. The ticket is used for the next authentication by the neighbor of the sink. The neighbor sinks verify the ticket and sends the updated one to the sink. The verification of the ticket is done using the authentication key inherited 'cluster key' in [9]-[10]. The main difference is that the key is used for broadcast communication in the cluster, while the key in our protocol is used for verifying the authentication ticket.

\section{Problems in The Smart Home}

Since the resident of the smart home does not consider the regularity arranging the devices such as TVs, DVDs, and microwaves, the regular distribution is not expected in real environments.

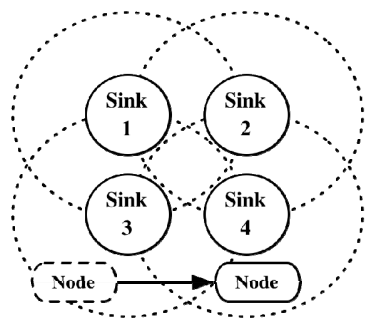

(a) Ideal Environments

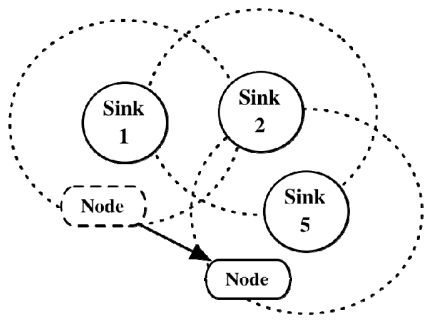

(b) Real Environments
Fig. 3 Sinks are regularly distributed in the ideal environments as in (a). However, in the real environments such as the smart home, the sinks may be distributed irregularly as in (b).

The previous protocol works well in the ideal environments as in Fig. 3 (a). However, the node may fail to be reauthenticated in case sinks are irregularly distributed. The node authenticated by $S_{1}$ may move and reconnected to $S_{5}$. However, $S_{5}$ is not the neighbor of $S_{l}$, the node cannot be reauthenticated as in Fig. 3 (b).

\section{IMPROVEMENT FOR SMART HOME}

In this section, we show the improvement of the previous protocol for the smart home. We introduce the concept of 'Neighbor Sink List (NSL)' in order to make our protocol be applicable in the real environments, and show the improvement with NSL.

\section{A. Neighbor Sink List}

When a sink finds the neighbor sinks, the sink stores the list of the neighbors. The neighbor sink list (NSL) of a sink $S_{I}$ is denoted as $N S L_{S_{1}}$, where $\mathrm{NSL}_{\mathrm{S}_{\mathrm{i}}}=S_{i}\left\|h\left(S_{j}\right)\right\| \ldots\left\|h\left(S_{j+k}\right)\right\| M$, and $M=M A C_{A K_{S_{i}}}\left(S_{i}\left\|h\left(S_{j}\right)\right\| \ldots \| h\left(S_{j+k}\right)\right)$.

S1

S5

\begin{tabular}{|c|c|}
\hline S10 & S11 \\
\hline S3 & $-\mathrm{S}_{2}$ \\
\hline S4 & $\mathrm{S}_{7}$ \\
\hline $\mathbf{S}_{2}$ & S8 \\
\hline S6 & S9 \\
\hline
\end{tabular}

Fig. $4 S_{5}$ finds that $S_{1}$ and $S_{5}$ have the common neighbor $S_{2}$ by checking NSL of $S_{1}$.

NSL is sent to the node during authentication process. When a node authenticated by $S_{1}$ is reconnected to $S_{5}$, the node sends $N S L_{S_{1}}$ to $S_{5}$. Although $S_{1}$ is not the neighbor of $S_{1}, S_{5}$ finds out that $S_{2}$ is the common neighbor of both $S_{1}$ and $S_{5}$ as in Fig. 4. 


\section{B. Improved Protocol}

1) Periodical Neighbor Discovery Procedure

$S_{l}$ periodically generates a random nonce $R_{0}$. $S_{l}$ also generates $u_{0}$ and $v_{0}$, where $u_{0}=E_{K_{S_{1}}}\left(R_{0} \| T S_{0}\right)$ and $v_{0}=M A C_{I K_{S_{1}}}\left(S_{1} \|\right.$ HELLO $\left.\| u_{0}\right)$. TS $S_{0}$ is timestamp. Then $S_{I}$ broadcasts $u_{0}$ and $v_{0}$ with HELLO. We have no change from the previous protocol.

\section{2) Establishing Neighbor Sink List}

Assume another sink $S_{2}$ receives HELLO message. $S_{2}$ checks the sender of HELLO whether $S_{1}$ is known or not. If $S_{2}$ already knows $S_{1}, S_{2}$ discards the message. Otherwise, $S_{2}$ requests the setting up the neighbor relationship as follows:

$S_{2}$ randomly selects $R_{1}$ and generates $u_{1}$ and $v_{l}$, where $u_{1}=E_{K_{S_{2}}}\left\{R_{1} \| u_{0}\right\}$ and $v_{1}=M A C_{I K_{S_{2}}}\left(S_{2}\|\mathrm{BS}\| S_{1}\left\|u_{1}\right\| v_{0}\right)$. After verifying $v_{l}$, BS decrypts $u_{l}$ and retrieves $R_{l}$ and $u_{0}$. Then, BS verifies $v_{0}$ and decrypts $u_{0}$. Finally, BS retrieves $R_{0}$ and $T S_{0}$, and then generates $u_{3}, u_{4}, v_{4}$, and $v_{3}$, where $u_{3}=E_{K_{S_{1}}}\left\{R_{1} \| h\left(T S_{0}\right)\right\} \quad, \quad v_{3}=M A C_{I K_{S 1}}\left(\mathrm{BS}\left\|S_{1}\right\| u_{3}\right) \quad$, $u_{4}=E_{K_{2}}\left\{R_{1} \| u_{3}\right\} \quad$ and $\quad v_{4}=M A C_{I K_{2}}\left(\mathrm{BS}\left\|S_{2}\right\| R_{1}\left\|u_{4}\right\| v_{3}\right)$. And then BS sends $u_{4}, v_{4}$, and $v_{3}$ to $S_{2}$. Then $S_{2}$ verifies $v_{4}$ and decrypts $u_{4}$, and retrieves $R_{1}$ and $u_{3} . S_{2}$ generates the encryption key $K_{S_{1} S_{2}}$ and the integrity key $I K_{S_{1} S_{2}}$ shared between $S_{1}$ and $S_{2}$, where $K_{S_{1} S_{2}}=\operatorname{KDF}\left(0\left\|R_{0}\right\| R_{1}\right)$ and $I K_{S_{1} S_{2}}=K D F\left(1\left\|R_{0}\right\| R_{1}\right) \quad$. Then $S_{2}$ generates $v_{5}$, where $v_{5}=M A C_{I K_{S_{1} S_{2}}}\left(S_{2}\left\|S_{1}\right\| R_{0} \| R_{1}\right)$, and sends $u_{3}, v_{3}$, and $v_{5}$ to $S_{l}$. After verifying $v_{3}, S_{1}$ decrypts $u_{3}$ and retrieves $R_{l} . S_{l}$ also generates $K_{S_{1} S_{2}}$ and $I K_{S_{1} S_{2}}$. Then $S_{1}$ verifies $v_{5}$. $S_{1}$ generates $v_{6}=M A C_{I K_{S_{1} S_{2}}}\left(S_{1}\left\|S_{2}\right\| A C K\left\|R_{0}\right\| R_{1}\right)$ and sends $v_{6}$ with ACK to $S_{2}$. $S_{2}$ verifies $v_{6}$ and shares pairwise keys $K_{S_{1} S_{2}}$ and $I K_{S_{1} S_{2}}$. As a result, $S_{1}$ and $S_{2}$ update their NSL.

\section{3) Distribution of Authentication Key}

After neighbor sinks are found, the sink $S_{l}$ may distribute the authentication key (AK). $S_{l}$ randomly selects two nonce $A S E E D_{S_{1}}$ and $R_{l}$. Then $s_{1}$ generates $u_{l}$ and $v_{l}$, where $u_{1}=E_{K_{S_{1} S_{2}}}\left\{A S E E D_{S_{1}} \| R_{1}\right\}$ and $v_{1}=M A C_{I K_{S_{1} S_{2}}}\left(S_{1}\left\|S_{2}\right\| u_{1}\right)$. After verifying $v_{l}, S_{2}$ decrypts $u_{l}$, and retrieves $A S E E D_{S_{1}}$ and $R_{l}$. Then $S_{2}$ generates $A K_{S_{1}}=K D F\left(0 \| A S E E D_{S_{1}}\right) \quad$ and $A I K_{S_{1}}=K D F\left(1 \| A S E E D_{S_{1}}\right) . S_{2}$ also generates $v_{2}$ using $A I K_{S_{1}}$, where $v_{2}=M A C_{A I K_{S_{1}}}\left(S_{2}\left\|S_{1}\right\| \mathrm{ACK} \| A R_{1}\right)$. Then $S_{1}$ verifies $v_{2}$.

\section{4) Initial Node Authentication}

Assume a node $N$ is firstly joining the sensor network. When $N$ receives HELLO of $S_{l}, N$ randomly selects $R_{l}$ and generates $u_{l}$ and $v_{l}$ and sends them to $S_{l}$, where $u_{1}=E_{K_{N}}\left\{R_{1}\left\|u_{0}\right\| v_{0}\right\}$ and $v_{1}=M A C_{I K_{N}}\left(N_{1}\left\|S_{1}\right\| u_{1}\right)$. Then, $S_{I}$ generates $v_{2}$, where $v_{2}=M A C_{I K_{S_{1}}}\left(S_{1}\|\mathrm{BS}\| N\left\|u_{1}\right\| v_{1}\right)$, and sends it to BS. After verifying $v_{2}$ and $v_{l}$, BS decrypts $u_{l}$, and retrieves $R_{0}, u_{0}$ and $v_{0}$. After verifying $v_{0}$, BS decrypts $u_{0}$, and retrieves $R_{0}$ and $T S$. BS checks the validity of $T S$ and generates $u_{3}, v_{3}, u_{4}$, and $v_{4}$, where $u_{3}=E_{K_{N}}\left\{R_{0}\right\}$, $v_{3}=M A C_{I K_{N}}\left(B S\|N\| S_{1} \| u_{3}\right) \quad, \quad u_{4}=E_{K_{S_{1}}}\left\{R_{1}\left\|u_{3}\right\| v_{3}\right\} \quad$ and $v_{4}=M A C_{I K_{S_{1}}}\left(B S\left\|S_{1}\right\| N\left\|R_{0}\right\| u_{4}\right)$.

After verifying $v_{4}, S_{l}$ decrypts $u_{4}$, and retrieves $R_{l}, u_{3}$ and $v_{3}$. Then $S_{1}$ generates $N K_{N}=\operatorname{KDF}\left(R_{0} \| R_{1}\right) . S_{l}$ generates authentication ticket $T=(t, w)$, where $t=E_{A K_{S_{1}}}\left\{T S\left\|R_{1}\right\| N K_{N}\right\}$ and $w=M A C_{A I K_{S_{1}}}(N \| t) . \quad S_{1}$ also generates $u_{5}$ and $v_{5}$, where $u_{5}=E_{N K_{N}}\left\{T S\|T\| N S L_{S_{1}}\right\}$ and $v_{5}=M A C_{N I K_{N}}\left(S_{1}\|N\| R_{0} \| u_{5}\right)$. $S_{l}$ sends $v_{3}, u_{5}$, and $v_{5}$ to $N$.

After verifying $v_{3}, N$ decrypts $u_{3}$ and retrieves $R_{0}$. Then $N$ also generates $N K_{N}$ and verifies $v_{5} . N$ decrypts $u_{5}$ and retrieves $T S, T$ and $N S L_{S_{1}} . N$ generates $v_{6}$, where $v_{6}=M A C_{N K_{N}}\left(N\left\|S_{1}\right\| A C K\left\|R_{0}\right\| R_{1}\right)$. $S_{l}$ verifies $v_{6}$.

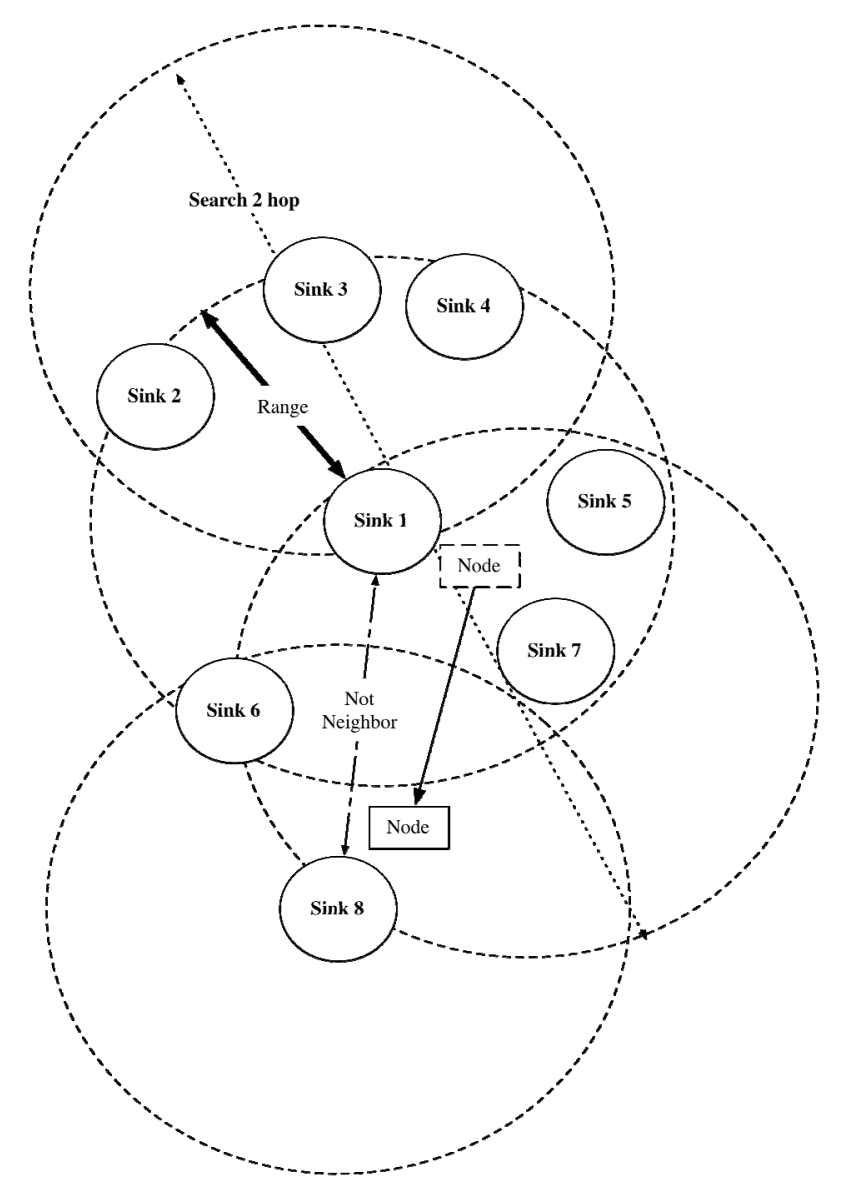

Fig. 5. When the node authenticated by $S_{1}$ is reconnected $S_{8}, S_{8}$ authenticates the node by finding the common neighbor $S_{6}$ comparing NSL of $S_{1}$ and $S_{8}$, and requesting the authentication of the node to $S_{6}$.

5) Node Re-authentication in Ideal Environments

Once the node $N$ is authenticated, $N$ can have the reduced overhead for the following authentication. Assume $N$ moves and receives HELLO from $S_{2} . N$ generates $v_{l}$, and sends $T, v_{l}$ and $N S L_{S_{1}}$ to $S_{2}$, where $v_{1}=M A C_{N I K_{N}}\left(N\left\|S_{2}\right\| T\left\|N S L_{S_{1}}\right\| v_{0}\right)$. Then $S_{2}$ check $N S L_{S_{1}}$ if $S_{1}$ is the neighbor of $S_{2}$.

When $S_{1}$ is the neighbor of $S_{2}, S_{2}$ verifies $T$ and decrypts $t$ using the authentication key $A K_{S_{1}} . S_{2}$ retrieves $R_{l}, N K_{N}$ and $T S$. Using $N K_{N}, S_{2}$ verifies $v_{l}$. Then $S_{2}$ generates new shared 
key $N K_{N}^{\prime}=K D F\left(R_{1} \| R_{0}\right)$, also generates the new authentication ticket $T^{\prime}=\left(t^{\prime}, w^{\prime}\right)$, where $t^{\prime}=E_{A K_{S_{2}}}\left\{R_{1} \| N K_{N}^{\prime}\right\} \quad$ and $w^{\prime}=M A C_{A I K_{S_{2}}}\left(N \| t^{\prime}\right) \quad . \quad S_{2}$ generates $\quad v_{2}=h\left(N K_{N}^{\prime} \| R_{0}\right) \quad$ and $u_{3}=E_{N K_{N}}\left\{R_{0}\left\|v_{2}\right\| T^{\prime} \| N S L_{S_{2}}\right\}, v_{3}=M A C_{N I K_{N}}\left(S_{2}\|N\| u_{3}\right)$.

After verifying $v_{3}, N$ decrypts $u_{3}$ and retrieves $R_{0}, v_{2}, T$ ' and $N S L_{S_{2}}$. Then $N$ generates $N K_{N}^{\prime}$ and verifies $v_{2}$. $N$ generates $v_{4}$, where $v_{4}=M A C_{N I K_{N}^{\prime}}\left(N\left\|S_{2}\right\| \mathrm{ACK}\left\|R_{0}\right\| R_{1}\right)$, and sends $v_{4}$ with ACK to $S_{2}$. After verifying $v_{4}, S_{2}$ authenticates $N$.

\section{6) Node Re-authentication in Real Environments}

In case the node $N$ that was authenticated by the sink $S_{l}$ is reconnected to other sink $S_{8}$ as in Fig, the node may fail to be authenticated in the previous scheme, since $S_{8}$ is not the neighbor sink of $S_{l}$. However, our improvement enables the efficient re-authentication of $N$.

When $S_{8}$ receives $N S L_{S_{1}}, S_{8}$ identify that $S_{1}$ is not the neighbor. Instead, $S_{8}$ finds that the neighbor sink $S_{6}$ is also the neighbor of $S_{1}$. (Refer Fig. 4.) Thus, $S_{8}$ sends the authentication ticket $T$ to $S_{6}$ and request verification, then $S_{6}$ verifies $T$ using $A K_{S_{1}}$ of $S_{I}$ and returns the results to $S_{8}$. With the results from $S_{6}, S_{8}$ generates $N K_{N}^{\prime}$ and $T$ '. The remaining follows the process in ideal environments.

\section{Analysis}

In this Section, we analyze our improved protocol with comparing the previous protocol. For the performance analysis, we compare the number of communication passes, the required message sizes, and the number of computation of the protocol. We do not count the overhead in the neighbor discovery procedure, since the node just ignores this procedure when the node receives HELLO from the sink that already authenticated the node.

\section{A. Communication Pass}

We compared the required number of communication passes with Fantacci et al.'s model [6], Ibriq and Mahgoub's model [4], and original model [2]. TABLE I shows the comparison of communication passes for node re-authentication, where $n$ denotes the number of nodes and $t$ denotes the number of sinks. Since nodes act as the authentication server (the base station) and the authenticator (the sink), all the communications in [6] are operated among nodes.

Comparison of required number of communication pass in initial authentication is as same as the previous models. In reauthentication of the nodes, Improved model requires 2 more communication for re-authentication in real environments than the original model, while it is still much efficient than [3]-[4].

TABLE I

COMPARISON OF COMMUNICATION PASS FOR RE-AUTHENTICATION

\begin{tabular}{|c|c|c|c|c|}
\hline & $\begin{array}{c}\text { Fantacci , } \\
\text { et al.'s [6] }\end{array}$ & $\begin{array}{c}\text { Ibriq } \text { et } \\
\text { al.'s [4] }\end{array}$ & $\begin{array}{c}\text { Previous } \\
\text { Model [2] }\end{array}$ & $\begin{array}{c}\text { Proposed } \\
\text { model }\end{array}$ \\
\hline Node & 2 & $2 \mathrm{n}$ & 2 & 2 \\
\hline Sink & $2 \mathrm{t}+1$ & $2 \mathrm{t}$ & 1 & $1(3)$ \\
\hline Base station & - & 2 & - & \\
\hline
\end{tabular}

\section{B. Message Size}

We compared Abraham and Ramanatha's model [3], [2] and [4] for the required message size for authentication. Based on the results in [3], we approximately compared the message sizes based on the message size with MAC size as 4 bytes, the time stamp as 8 bytes, nonce as 8 bytes, and key size as 16 bytes. We also set the source and target IDs as 1 byte, respectively.

TABLE II

COMPARISON OF MESSAGE SIZE FOR INITIAL AUTHENTICATION (BYTES)

\begin{tabular}{|c|c|c|c|c|}
\hline & $\begin{array}{c}\text { Abraham } \\
\text { 's model } \\
{[3]}\end{array}$ & $\begin{array}{c}\text { Ibriq and } \\
\text { Mahgoub's } \\
\text { model [4] }\end{array}$ & $\begin{array}{c}\text { Previous } \\
\text { Model } \\
{[2]}\end{array}$ & $\begin{array}{c}\text { Proposed } \\
\text { Model }\end{array}$ \\
\hline Node to Sink & 46 & 68 & 56 & 56 \\
\hline Sink to Sink & 70 & 76 & 62 & 62 \\
\hline $\begin{array}{c}\text { Sink to Base } \\
\text { station }\end{array}$ & 70 & 76 & 62 & 62 \\
\hline $\begin{array}{c}\text { Base station to } \\
\text { Node }\end{array}$ & 92 & 188 & 192 & 204 \\
\hline $\begin{array}{c}\text { Total message } \\
\text { size }\end{array}$ & 278 & 408 & 314 & 326 \\
\hline
\end{tabular}

TABLE II and III show the message sizes in initial authentication and the message sizes in re-authentication with 2 hops between sink and base station, respectively. TABLE II shows that the performance for the initial authentication is similar to other protocols. In initial authentication (Phase 3), Abraham and Ramanatha's model [3] showed the best result that 30 bytes less message sizes than our protocol. However, as the TABLE III shows, our protocol achieves about a third overall message sizes than other protocols. Even we increase the size of each parameter, our protocol is still much efficient than any other protocols in node re-authentication.

TABLE III

COMPARISON OF MESSAGE SIZE FOR RE-AUTHENTICATION (BYTES)

\begin{tabular}{|c|c|c|c|c|}
\hline & $\begin{array}{c}\text { Abraham's } \\
\text { model [3] }\end{array}$ & $\begin{array}{c}\text { Ibriq and } \\
\text { Mahgoub's } \\
\text { model [4] }\end{array}$ & $\begin{array}{c}\text { Previous } \\
\text { model } \\
\text { [2] }\end{array}$ & $\begin{array}{c}\text { Proposed } \\
\text { model }\end{array}$ \\
\hline Node to Sink & 46 & 68 & 44 & 56 \\
\hline Sink to Sink & 70 & 76 & & - \\
\hline $\begin{array}{c}\text { Sink to Base } \\
\text { station }\end{array}$ & 70 & 76 & 64 & 76 \\
\hline $\begin{array}{c}\text { Base station } \\
\text { to Node }\end{array}$ & 92 & 188 & 108 & 132 \\
\hline $\begin{array}{c}\text { Total } \\
\text { message size }\end{array}$ & 278 & 408 & & \\
\hline
\end{tabular}

Fig. 6 shows the comparison of our improved model with the previous models. While the message cost is increasing with the longer hop distance in the static models [3]-[4], the original model [2] and the improved model have the constant cost.

Fig. 7 shows the comparison of the proposed protocols in several environments. The result of initial authentication shows the increasing cost depends on the hop distance. The reauthentication cases show the constant result although overall cost increases depending on the rate that the sink is not the neighbor of the former sink. 


\section{Security Analysis}

Since the proposed protocol improves the previous protocol [2], most security features such as confidentiality, key freshness, and node/sink resiliency are inherited. Thus, we only concentrated on the analysis of the changes.

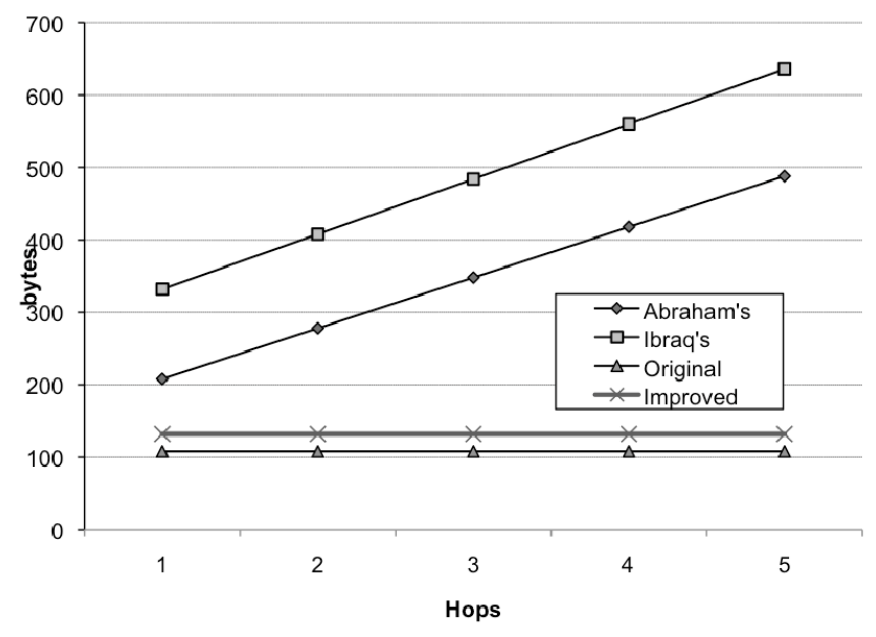

Fig. 6 Comparison of message sizes with static models [3]-[4], previous model [2] and improved model per hop distance between a sink and a base station.

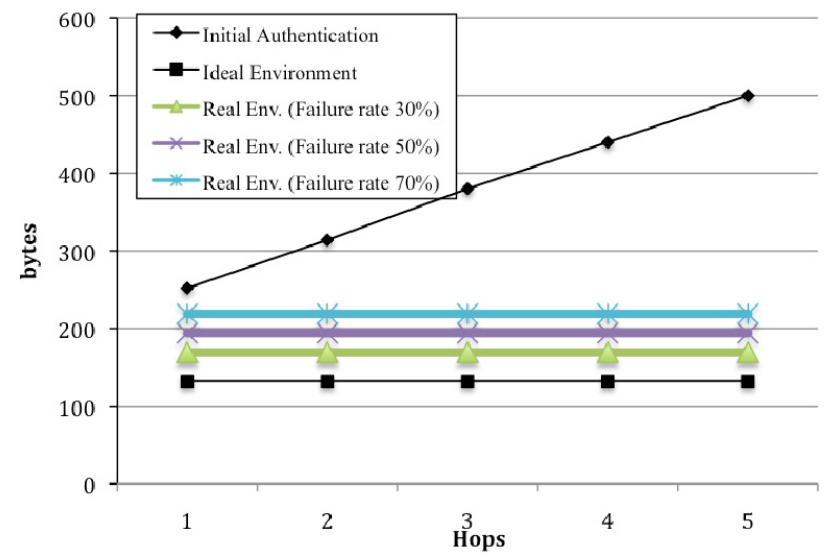

Fig. 7 Communication cost for proposed protocol. Depending on the failure rate, the cost increases, but still the cost shows the constant when the hop distance increases.

\section{1) Re-authentication using Neighbor Sink List}

After a node $N$ is initially authenticated by a $\operatorname{sink} S_{I}$ in phase 3 , the node receives the authentication ticket $T$ and $v_{l}$. When $N$ moves and requests re-authentication to the neighbor sink $S_{8}$, $S_{8}$ may fail to verify $T$ since $S_{l}$ is not the neighbor. However, $S_{8}$ and $S_{1}$ have the common neighbor $S 2$, and the authentication key of $S_{1}, A K_{S_{1}}$ is shared to $S_{2}$. Thus, with help of $S_{2}, S_{8}$ can authenticate $N$ and exchange the key. In the reauthentication phase, the base station is not involved.

\section{2) Untraceability using Neighbor Sink List}

When $S_{8}$ authenticates $N, S_{2}$ involves in the protocol. However, the role of $S_{2}$ is just verifying and decrypting $T$. Therefore $S_{2}$ cannot predict $N$ 's next movement.
3) Security against known attacks

The sinkhole attack against our protocol fails without knowing the keys. An adversary $A$ may capture the authentication ticket $T$ that $N$ initially sent to $S_{2}$, and $A$ send $T$ to $S_{2}$ or other sink $S_{5}$ that is also a neighbor sink of $S_{l}$. However, $A$ fails in such attack without knowing $A K_{S_{1}}$. Wormhole attack on our protocol fails since the adversary cannot send the confirmation message. Spoofed, altered or replayed routing information attack also fails with our knowing encrypted nonce in our protocol. To succeed in the replay attack, the adversary has to be able to reuse the intercepted packet. We don't consider relaying through the attackers as successful attack. Sybil attacks also fails from verification of identity of nodes through sinks and the base station. And for HELLO flood attacks, we can apply the global key shared to all entities in the network that many researches such as [4], [9], [10] used for the efficient message broadcast and DoS attack protection.

\section{Conclusion}

Recently, Smart Home is emerging and extending rapidly as new converged paradigms including fusion \& convergence, smart grid, machine-to-machine, and peer-to-peer pervasive computing to provide fully always-connected services with mobility. Thus, it is very important to support dynamic topology among various CE and IT devices. Specifically, the failure of the node re-authentication can be occurred frequently because the previous works only considered the environment that the sensors are regularly distributed ideally.

In this paper, our proposed improvement enables the efficient node re-authentication and key exchange even when the sensors are irregularly distributed to the smart home and WPAN for supporting various convergence services. In order to verify the proposed approach, we perform three kinds of validation according to communication pass, message size, and security analysis. From the analysis, we can say that our improvement guarantees the longer lifetime of Smart Home Devices and WPAN while providing security solutions.

In future work we will deploy the proposed approach to real Smart home environments and confirm the authentication operations for supporting NSL.

\section{REFERENCES}

[1] H. Chan, A. Perrig, and D. Song, "Random Key Predistribution Schemes for Sensor Networks", in IEEE Symposium on Security and Privacy, Berkeley, California, pp. 197-213, 2003.

[2] K. Han, K, Kim, and T. Shon, "Untraceable Mobile Node Authentication in WSN," accepted to Sensors 2010 (ISSN 1424-8220; CODEN: SENSC9), Molecular Diversity Preservation International (MDPI), 2010

[3] J. Abraham, and K.S. Ramanatha, "An Efficient Protocol for Authentication and Initial Shared Key Establishment in Clustered Wireless Sensor Networks," Proceeding of Third IFIP/IEEE International Conference on Wireless and Optical Communications Networks, 2006.

[4] J. Ibriq, and I. Mahgoub, "A Hierarchical Key Establishment Scheme for Wireless Sensor Networks," Proceedings of 21st International Conference on Advanced Networking and applications (AINA'07), 2007, pp. 210-219.

[5] L. Eschenauer, and V. Gligor, "A key management scheme for distributed sensor networks," in Proceedings of the 9th ACM conference on Computer and Communications Security (CCS), Washington. DC. USA 2002, pp. 41-47. 
[6] R. Fantacci, F. Chiti, and L. Maccari, "Fast distributed bi-directional authentication for wireless sensor networks", Security and Communication Networks, John Wiley \& Sons, pp. 17-24, 2008.

[7] S. Das, H. Liu, A. Kamath, A. Nayak, and I. Stojmenovic, "Localized Movement Control For Fault Tolerance of Mobile Robot Networks," in IFIP International Federation for Information Processing, Wireless Sensor and Actor Networks, eds. L. Orozco-Barbosa, Olivares, T., Casado, R., Bermudez, A., (Boston:Springer) 2007, 248.

[8] S. S. Krishnakumar, and R. T. Abler, "Intelligent Actor Mobility in Wireless Sensor and Actor Networks," in IFIP International Federation for Information Processing, Wireless Sensor and Actor Networks, eds. L. Orozco-Barbosa, Olivares, T., Casado, R., Bermudez, A., (Boston:Springer) 2007, pp. 13-22.

[9] S. Zhu, S. Setia, and S. Jajodia, "LEAP: efficient security mechanisms for largescale distributed sensor networks," In CCS '03: Proceedings of the 10th ACM conference on Computer and communications security. ACM: New York, NY, USA, 2003, pp. 62-72.

[10] S. Zhu, S. Setia, and S. Jajodia, "LEAP+: Efficient security mechanisms for large-scale distributed sensor networks," ACM Trans. Sen. Netw. 2006, 2, 500528.

[11] W. C. Craig, "Zigbee:Wireless Control That Simply Works," Zigbee Alliance 2005.

[12] W. Du, J. Deng, Y. S. Han, and P. K. Varshney, "A Pairwise Key Predistribution Scheme for Wireless Sensor Networks," in Proceedings of the 10th ACM conference on Computer and Communications Security (CCS), Washington. DC. USA 2003, pp. 42-51.

[13] E. Callaway, P. Gorday, and L. Hester, "Home Networking with IEEE 802.15.4: A Developing Standard for Low-Rate Wireless Personal Area Networks", IEEE Communications Magazine, vol. 40, no. 8, pp. 70-77, 2002.

[14] G. K., S. Yang, F. Yao, and X. Lu, "A zigbee-based home automation system", IEEE Transactions on Consumer Electronics, vol. 55, no. 2, pp 422 - 430, 2009.

\section{BIOGRAPHIES}

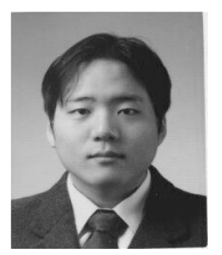

Kyusuk Han received the B.S. degree in Mechanical Engineering from Hongik University, Korea and the M.S. degree in Computer Science from Information and Communications University, Korea, respectively in 2001 and 2004. He is presently Doctorate course student in School of Engineering, KAIST, Korea. His interests are in cryptography and information security.

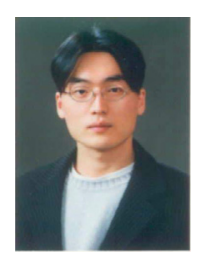

Taeshik Shon (M'10) is a senior engineer in the Convergence Solution Team, DMC R\&D Center of Samsung Electronics Co., Ltd. He received his Ph.D. degree in Information Security from Korea University, Seoul, Korea, 2005 and his M.S. and B.S. degree in computer engineering from Ajou University, Suwon, Korea, 2000 and 2002, respectively. While he was working toward his Ph.D. degree, he was awarded a KOSEF scholarship to be a research scholar in the Digital Technology Center, University of Minnesota, Minneapolis, USA, from February 2004 to February 2005. He was awarded the Gold Prize for the Sixth Information Security Best Paper Award from the Korea Information Security Agency in 2003, the Honorable Prize for the 24th Student Best Paper Award from Microsoft-KISS, 2005, the Bronze Prize for the Samsung Best Paper Award, 2006, and the Second Level of TRIZ Specialist certification in compliance with the International TRIZ Association requirements, 2008. He is also serving as an editorial staff and review committee of the Journal of The Korea Institute of Information Security and Cryptology, IAENG International Journal of Computer Science, and other journals. His research interests include Mobile/Wireless Network Security, WPAN/WSN Network Security, network intrusion detection systems, and machine learning.

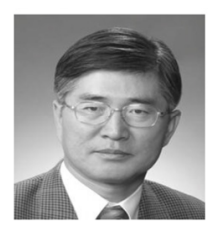

Kwangjo Kim received the B.S and M.S. degree of Electronic Engineering in Yonsei University, Korea, and $\mathrm{Ph} . \mathrm{D}$ of Div. of Electrical and Computer Engineering in Yokohama National University, Japan. Currently he is Professor at School of Computer Science in KAIST, Korea. He is also the president of Korean institute on Information Security and Cryptography. 